\title{
CORRECTION
}

\section{Correction to: Assessing the Safety of COVID-19 Vaccines: A Primer}

\author{
Helen Petousis-Harris ${ }^{1}$
}

Published online: 16 February 2021

(c) Springer Nature Switzerland AG 2021

\section{Correction to: Drug Safety (2020) \\ https://doi.org/10.1007/s40264-020-01002-6}

The Conflicts of interest/competing interests section, which previously read:

"Helen Petousis-Harris is engaged in a global collaboration that aims to assess vaccine safety and risk benefit"

should read:

"Helen Petousis-Harris is engaged in a global collaboration that aims to assess vaccine safety and risk benefit. She was a member, and a former chair of, the Global Advisory Committee on Vaccine Safety. She is also a current member of the Brighton Collaboration's Science Board."

The original article can be found online at https://doi.org/10.1007/ s40264-020-01002-6.

Helen Petousis-Harris

h.petousis-harris@auckland.ac.nz

1 Department of General Practice and Primary Health Care, Faculty of Medical and Health Sciences, University of Auckland, Auckland, New Zealand 